\title{
Erratum to: Comparison of a drug-eluting balloon first and then bare metal stent with a drug-eluting stent for treatment of de novo lesions: study protocol of a randomized controlled trial
}

\author{
Sang-Don Park ${ }^{\dagger}$, Chang-Hwan Yoon ${ }^{\dagger}$, Il-Young Oh, Jung-Won Suh, Young-Suk Cho, Tae-Jin Youn, \\ Dong-Ju Choi and In-Ho Chae
}

Unfortunately, the original version of this article [1] contained an error. The correct paragraph can be found below:

The calculation of number of patients was not correct. It was wrote the non-inferiority margin as $0.1 \mathrm{~mm}$. But it was $0.19 \mathrm{~mm}$ in an original document. Non-inferiority margin in previous coronary intervention studies has been set between $0.1 \sim 0.2 \mathrm{~mm}$. The $0.19 \mathrm{~mm}$ is not irrelevant in view of the current trial standard.

Received: 11 January 2017 Accepted: 11 January 2017

Published online: 23 May 2017

\section{Reference}

1. Park S, Yoon C, Oh I, Suh J, Cho Y, Youn T, Choi D, Chae I. Comparison of a

drug-eluting balloon first and then bare metal stent with a drug-eluting

stent for treatment of de novo lesions: study protocol of a randomized

controlled trial. Trials. 2013;14:38. doi:10.1186/1745-6215-14-38.

\footnotetext{
* Correspondence: ihchae@snu.ac.kr

${ }^{\dagger}$ Equal contributors

Division of Cardiology, Department of Internal Medicine, Seoul National

University Bundang Hospital, 82 Gumi-ro, 173 Bein-gil, Bundang-gu,

Seongnam-si, Gyeonggi-do 463-707, Republic of Korea
} 